\title{
Avaliação de Sistema de Leitura Portátil (SLP) para Baixa Visão Desenvolvido no Brasil
}

\author{
Evaluation of System Portable Reading (SPR) \\ for Low Vision Developed in Brazil
}

Vagner Rogério dos Santos ${ }^{1}$, Eliana Cunha Lima ${ }^{2}$, Adriana Berezovsky³, Paulo Schor ${ }^{4}$, Ricardo Uras ${ }^{5}$, Rubens Belfort Junior ${ }^{6}$

\section{RESUMO}

Objetivo: A proposta deste estudo é avaliar a eficácia e eficiência em uma série de casos do protótipo de um equipamento nacional de magnificação para leitura. Métodos: Participaram deste estudo 30 pacientes na faixa etária entre 9 e 80 anos (17 do sexo masculino). Foi desenvolvido um aparelho portátil, patenteado pela UNIFESP (PI\#020050145260), com um sistema de captura de imagens acoplado a um monitor de 5,6 polegadas proporcionando um aumento de $15 \mathrm{X}$. Foram analisadas a eficácia da acuidade visual e a eficiência de leitura após a utilização do protótipo proposto. Resultados: Seis pacientes $(20 \%)$ apresentaram AV $8 \mathrm{M}, 12$ pacientes $(40 \%)$ apresentaram AV 6M, 7 pacientes $(23,3 \%)$ apresentaram $5 \mathrm{M}, 5$ pacientes $(16,7 \%)$ apresentaram $4 \mathrm{M}$. A média de acuidade visual antes da utilização do SLP medida pela tabela LHNV-1 $\log$ MAR foi de $5,75 \mathrm{M}$ e após a utilização $100 \%$ dos pacientes atingiram a eficácia de AV J1.Conclusão: O protótipo do SLP mostrou-se um recurso alternativo no processo de inclusão social das pessoas com baixa visão com diferentes níveis de resíduo visual. Também pode proporcionar incentivo psicológico, permitir conforto, mobilidade e independência àqueles que necessitam de uma leitura mais prolongada e maior distância de trabalho.

Descritores: Baixa visão/reabilitação; Desenho de equipamento; Engenharia biomédica; Leitura; Qualidade de vida; Brasil

'Tecnólogo em Mecânica de Precisão, Preceptor do Curso de Tecnologia Oftálmica da Universidade Federal de São Paulo UNIFESP - São Paulo (SP), Brasil.

${ }^{2}$ Ortoptista, Coordenadora do Setor de Reabilitação Visual da Fundação Dorina Nowill para Cegos. São Paulo (SP), Brasil.

${ }^{3}$ Doutora, Professora Adjunto do Departamento de Oftalmologia da Universidade Federal de São Paulo - UNIFESP - São Paulo (SP), Brasil.

${ }^{4}$ Livre-docente, Professor Afiliado e Chefe do Setor de Bioengenharia do Departamento de Oftalmologia da Universidade Federal de São Paulo - UNIFESP - São Paulo (SP), Brasil.

${ }_{5}^{5}$ Professor Adjunto do Departamento de Oftalmologia da Universidade Federal de São Paulo - UNIFESP - São Paulo (SP), Brasil.

${ }^{6}$ Livre-docente, Professor Titular do Departamento de Oftalmologia da Universidade Federal de São Paulo - UNIFESP - São Paulo (SP), Brasil.

Recebido para publicação em: 11/8/2008 - Aceito para publicação em 8/6/2009 


\section{INTRODUÇÃO}

A Organização Mundial de Saúde (OMS) estimou em 2002 a existência de 161 milhões de deficientes visuais na população mundial, dos quais 37 milhões eram cegos e 124 milhões com visão subnormal.

De acordo com a "International statistical classification of diseases, injuries and causes of death", décima revisão (ICD-10), define-se visão subnormal ou baixa visão quando a acuidade visual é inferior a 6/ 18 , mas igual ou melhor do que $3 / 60$, ou apresentar uma redução de campo visual, de tal forma que ele se torne menor do que $20^{\circ}$ no melhor olho e com a melhor correção possível ${ }^{(1)}$.

Esses indivíduos apresentam dificuldades de leitura e aprendizado, necessitando de adaptações ambientais em escolas e bibliotecas e da utilização de recursos ópticos, não ópticos e/ou eletrônicos que proporcionem melhor aproveitamento do seu resíduo visual. Tal necessidade pode caracterizar-se como um problema de saúde pública, pois, nos países de terceiro mundo e em desenvolvimento, a falta de verbas públicas é notória, o que inviabiliza a compra de recursos especiais por escolas e bibliotecas públicas ${ }^{(2)}$.

A inclusão de pessoas com deficiência é de suma importância nas sociedades organizadas. Para tal, a disponibilidade de ajudas técnicas que possibilitam mobilidade, independência, acesso à informação e ao estudo é um fator de grande impacto para acelerar a inclusão social e cultural das pessoas com deficiência visual.

No âmbito das ajudas técnicas, vale ressaltar que, com freqüência, sua utilização pelos estudantes gera problemas de ordem comportamental. Sabe-se que principalmente na adolescência a aceitação do grupo é fator determinante do comportamento de jovens. Usar um recurso óptico diferente dos óculos comuns, que não apresenta uma estética desejável, ou mesmo material escolar diferente dos demais colegas, pode causar problemas de auto-estima. Em alguns casos, alunos preferem não utilizar o auxílio, por referir problemas de rejeição oriundos de motivos estéticos e emoções negativas. ${ }^{(2,3)}$ A dificuldade na leitura impacta desfavoravelmente na educação, fator primordial para a inclusão, pois a leitura tem importante papel nas interações sociais e nas atividades produtivas, deixando à margem do sistema produtivo e cultural indivíduos capazes de produzir riquezas e de contribuir para o desenvolvimento da sociedade impondo um alto impacto ao custo social ${ }^{(4-6)}$.

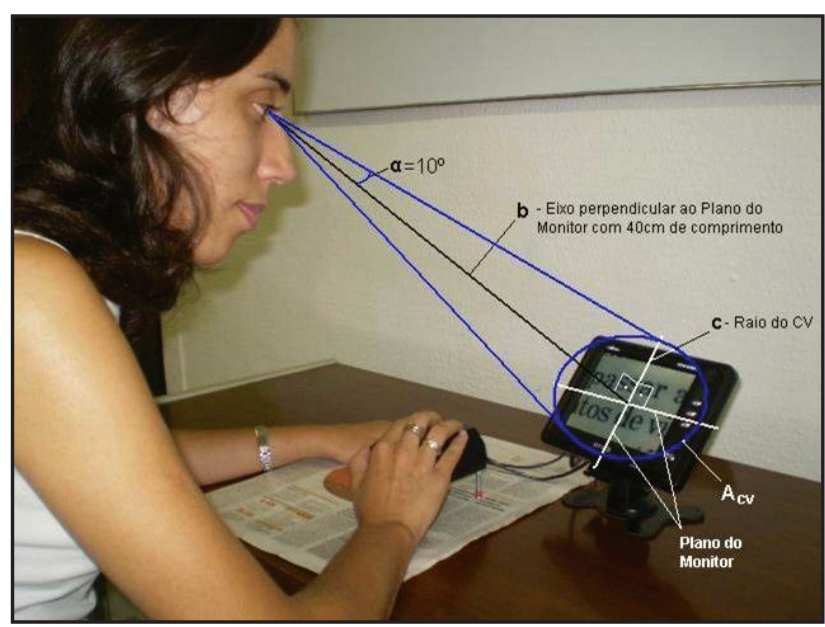

Figura 1 - Paciente JM (22 anos), AV 4.0M utilizando o SLP. Cálculo dos parâmetros do monitor baseado na equação bxtg $\alpha=c$. Variáveis: $b=$ eixo perpendicular ao plano do monitor correspondente a distância de leitura de $40 \mathrm{~cm} ; \operatorname{tg} \alpha=$ tangente do ângulo alfa de visão, $\alpha=$ ângulo de visão de $10^{\circ}, \mathrm{c}=$ raio do campo visual (CV). Obtém-se: $40 x \operatorname{xtg} \alpha 10^{\circ}=\mathrm{c} \leftrightarrow 7,05 \mathrm{~cm}$. Utilizando a equação $\mathrm{Acv}=\Pi$ x c $\mathrm{c}^{2}$ Variáveis: constante $\mathrm{pi} \rightarrow \Pi=3,14 \mathrm{e} \mathrm{c}=$ Raio do Campo Visual temos: $3,14 \times 7,05^{2} \leftrightarrow 156,202 \mathrm{~cm}^{2}$ que estabeleceu o critério de escolha do tamanho do monitor

A proposta deste estudo é avaliar a eficácia e eficiência do protótipo de um aparelho nacional para leitura e estudo de pacientes com baixa visão, patenteado pela UNIFESP com Patente Nacional

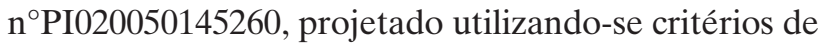
desenvolvimento e de usabilidade.

\section{Métodos}

Esta pesquisa segue os princípios básicos contidos na declaração de Helsinque e aprovada pelo Comitê de Ética Médica em Pesquisa do Hospital São Paulo/ UNIFESP.

Foi realizado estudo no Setor de Reabilitação Visual da Fundação Dorina Nowill para Cegos, no período de 19 de março a 14 de junho de 2007.

Os critérios de inclusão estabelecidos foram: indivíduos com diagnóstico prévio de visão subnormal pela definição da OMS, alfabetizados e anuência e assinatura do consentimento livre esclarecido.

Participaram 30 pacientes na faixa etária de 9 a 80 anos, sendo 17 do sexo masculino. Foram excluídos do estudo os pacientes com doenças neurológicas que pudessem afetar a coordenação motora.

Equipamento: Foi desenvolvido um sistema de captura óptico utilizando-se uma câmera "ChargeCoupled Device" (CCD) preto e branco, possibilitando um maior controle do contraste, com lente te- 


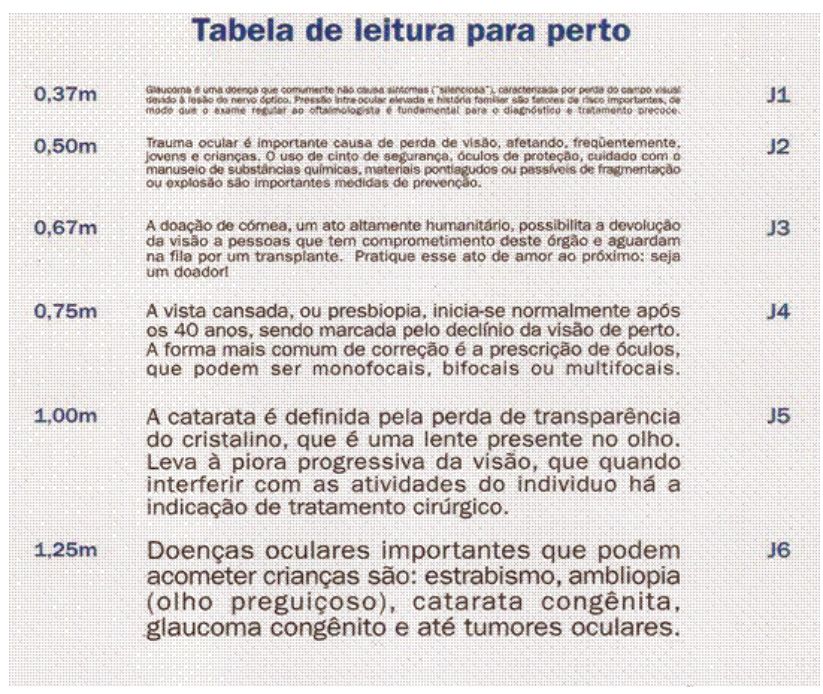

Figura 2: Tabela de leitura para perto Jaeger utilizada para avaliação da eficiência visual

lescópica de distância focal de $8 \mathrm{~cm}$. A este sistema de captura de imagens foi acoplado, por meio de sistema eletrônico um monitor de "Thin-film Transistor" (TFT) 5,6 polegadas colorido com resolução de 800 x 600 pixels. Para alimentação elétrica do SLP, utilizaram-se fontes de corrente contínua bivolt para uso em rede elétrica, garantindo a mobilidade do equipamento.

Os parâmetros utilizados para escolha do monitor foram estabelecidos mediante o cálculo da área do campo visual (Acv) baseado na equação: bx $\operatorname{tg} \alpha=c$. Onde as variáveis: $\mathrm{b}=$ eixo perpendicular ao plano do monitor correspondente à distância de leitura de $40 \mathrm{~cm} ; \operatorname{tg} \alpha=$ tangente do ângulo alfa de visão, $\alpha=$ ângulo de visão de $10^{\circ}$ e c= Raio do Campo Visual (CV), obtém-se: $40 \mathrm{x} \operatorname{tg} \alpha 10^{\circ}=\mathrm{c} \leftrightarrow 7,05 \mathrm{~cm}$.

$\mathrm{O}$ cálculo da área do campo visual basea-se na equação $\mathrm{Acv}=\Pi \mathrm{Xc}^{2}$. onde as variáveis: Constante $(\mathrm{pi}) \rightarrow \Pi$ $=3,14$ e c é o raio do campo visual.

Portanto $\mathrm{Acv}=\Pi \mathrm{X} \mathrm{c}^{2}$ temos: $3,14 \times 7,05^{2}=$ $156,202 \mathrm{~cm}^{2}$ que determinou a escolha do tamanho do monitor que fica inscrito nesta área (Figura 1).

Para atender as recomendações de usabilidade da norma ABNT NBR 9050, foi escolhido um monitor que permitisse o ajuste de sua inclinação, possibilitando ao usuário manter o eixo visual (b) sempre perpendicular ao plano de leitura do monitor, favorecendo uma melhor posição de leitura, e evitando cansaço físico e o surgimento de reflexo luminoso na tela do monitor.

A equação $\mathrm{M}=\mathrm{X}$ x Y foi utilizada para o cálculo do aumento de $15 \mathrm{X}$ do sistema óptico SLP ${ }^{(7)}$. sendo:

$\mathrm{X}=$ Tamanho do caractere em $\mathrm{cm}$

$$
\begin{aligned}
& X=\frac{\text { Letra do texto Lido }}{\text { Optotipo avaliado na AV }} \mathrm{cm} \\
& Y=\text { relação da distância de leitura } \\
& \mathrm{Y}=\frac{25}{40} \mathrm{~cm} \quad \begin{array}{l}
\text { Onde: } \\
25 \text { é uma constante em } \mathrm{cm} \\
40 \text { é a distância de leitura em } \mathrm{cm}
\end{array}
\end{aligned}
$$

A distância de $25 \mathrm{~cm}$ é uma constante, e é a distância mínima que possibilita conforto visual para leitura em circuito fechado de televisão (CCTV).

O peso do SLP é de aproximadamente $600 \mathrm{~g}$, o que permite melhor mobilidade ao paciente com deficiência visual.

Procedimento: Foram analisadas a eficácia e a eficiência visual do equipamento. Define-se eficácia como uma medida quantitativa do valor da acuidade visual e eficiência como a capacidade de discriminar e compreender o texto lido. Para o teste de eficácia da AV foi utilizada a tabela "The Lighthouse Near Acuity Test - LHNV-1", a $40 \mathrm{~cm}$, binocularmente com a correção óptica e iluminação padronizada de 80 candelas $/ \mathrm{m}^{2}$.

Em seguida, a mensuração foi realizada com o SLP sob as mesmas condições anteriormente descritas.

Foi utilizada para avaliação de eficiência visual a Tabela de leitura para perto Jaeger que contém frases equivalentes aos optotipos J1 a J6 (Figura 2).

\section{Resultados}

A tabela 1 mostra a distribuição das acuidades visuais antes e depois da utilização do recurso de magnificação SLP. Seis pacientes $(20 \%)$ apresentaram AV 8M, 12 pacientes (40\%) apresentaram AV 6,0 M, 7 pacientes (23,3\% ) apresentaram AV 5M, 5 pacientes $(16,7 \%)$ apresentaram $4 \mathrm{M}$.

A média de acuidade visual antes da utilização do SLP medida pela tabela LHNV-1 logMAR foi de $5,75 \mathrm{M}$ e após a utilização $100 \%$ dos pacientes atingiram a eficácia de AV J1.

\section{Discussão}

A escolha dos recursos mais adequados é uma das questões mais importantes no processo de reabilitação visual e inclusão social dos pacientes com baixa visão. Os recursos ópticos atualmente encontrados no mercado apresentam desvantagens significativas no uso diário. As lupas manuais, os óculos esfero-prismáticos e de 
Tabela 1

Análise descritiva da amostra em relação à idade, ocupação, patologia ocular e eficácia de leitura

\begin{tabular}{|c|c|c|c|c|c|c|c|}
\hline & \multirow[b]{2}{*}{ Paciente } & \multirow[b]{2}{*}{$\begin{array}{l}\text { Idade } \\
\text { (anos) }\end{array}$} & \multirow[b]{2}{*}{ Ocupação } & \multirow[b]{2}{*}{ Patologia ocular } & \multicolumn{3}{|c|}{ Eficácia Visual } \\
\hline & & & & & $\begin{array}{c}\text { AV AO } \\
\text { Apresentada } \\
\text { antes do } \\
\text { uso do SLP }\end{array}$ & $\begin{array}{c}\text { AV AO } \\
\text { com o } \\
\text { uso do } \\
\text { SLP } \\
\text { Tab LVRC }\end{array}$ & $\begin{array}{c}\text { AV AO } \\
\text { com o } \\
\text { uso do } \\
\text { SLP } \\
\text { Tab.Jaegger }\end{array}$ \\
\hline 1 & M.K.N & 58 & Aposentada & Glaucoma & $8.0 \mathrm{M}$ & $0,37 \mathrm{M}$ & $\mathrm{J} 1$ \\
\hline 2 & J.S & 24 & Universitário & Ceratocone & $5.0 \mathrm{M}$ & $0,37 \mathrm{M}$ & $\mathrm{J} 1$ \\
\hline 3 & J.R.S & 65 & Químico aposentado & $\begin{array}{l}\text { Degeneração macular } \\
\text { Relacionada à idade }\end{array}$ & $5.0 \mathrm{M}$ & $0,37 \mathrm{M}$ & $\mathrm{J} 1$ \\
\hline 4 & L.M & 70 & Aposentado & DMRI & $8.0 \mathrm{M}$ & $0,37 \mathrm{M}$ & $\mathrm{J} 1$ \\
\hline 5 & A.A.S & 62 & Aposentada & Alta Miopia & $6.0 \mathrm{M}$ & $0,37 \mathrm{M}$ & $\mathrm{J} 1$ \\
\hline 6 & A.F.K & 57 & Aposentada & Retinose pigmentária & $6.0 \mathrm{M}$ & $0,37 \mathrm{M}$ & $\mathrm{J} 1$ \\
\hline 7 & E.G.A & 45 & Aposentado & Traumatismo ocular & $6.0 \mathrm{M}$ & $0,37 \mathrm{M}$ & $\mathrm{J} 1$ \\
\hline 8 & L.M.S & 24 & $\begin{array}{l}\text { Desempregado/ } \\
\text { Ensino Médio Completo }\end{array}$ & $\begin{array}{l}\text { Alta miopia + Toxoplasmose } \\
\text { ocular congênita }\end{array}$ & $5.0 \mathrm{M}$ & $0,37 \mathrm{M}$ & $\mathrm{J} 1$ \\
\hline 9 & M.A. & 22 & Vestibulando & Coloboma de íris e córnea & $5.0 \mathrm{M}$ & $0,37 \mathrm{M}$ & $\mathrm{J} 1$ \\
\hline 10 & C.T.R & 23 & Universitário & Retinose pigmentária & $6.0 \mathrm{M}$ & $0,37 \mathrm{M}$ & $\mathrm{J} 1$ \\
\hline 11 & J.M & 22 & Universitária & Retinopatia da prematuridade & $4.0 \mathrm{M}$ & $0,37 \mathrm{M}$ & $\mathrm{J} 1$ \\
\hline 12 & M.L.S & 71 & Aposentada & Glaucoma & $4.0 \mathrm{M}$ & $0,37 \mathrm{M}$ & $\mathrm{J} 1$ \\
\hline 13 & D.M & 43 & Metalúrgico-aposentado & Stargardt & $5.0 \mathrm{M}$ & $0,37 \mathrm{M}$ & $\mathrm{J} 1$ \\
\hline 14 & O.B.A & 37 & Cantora & Catarata congênita & $6.0 \mathrm{M}$ & $0,37 \mathrm{M}$ & $\mathrm{J} 1$ \\
\hline 15 & T.T.S & 12 & $\begin{array}{l}\text { Estudante Ensino } \\
\text { Fundamental }\end{array}$ & Albinismo óculo cutâneo & $6.0 \mathrm{M}$ & $0,37 \mathrm{M}$ & $\mathrm{J} 1$ \\
\hline 16 & L.P & 10 & $\begin{array}{l}\text { Estudante Ensino } \\
\text { Fundamental }\end{array}$ & Retinoblastoma & $6.0 \mathrm{M}$ & $0,37 \mathrm{M}$ & $\mathrm{J} 1$ \\
\hline 17 & R.B.F & 39 & Estudante Ensino Médio & Albinismo óculo cutâneo & $4.0 \mathrm{M}$ & $0,37 \mathrm{M}$ & $\mathrm{J} 1$ \\
\hline 18 & R.T.S & 9 & $\begin{array}{l}\text { Estudante Ensino } \\
\text { Fundamental }\end{array}$ & Albinismo óculo cutâneo & $4.0 \mathrm{M}$ & $0,37 \mathrm{M}$ & $\mathrm{J} 1$ \\
\hline 19 & Z.L.T & 67 & Músico & Glaucoma congênito & $5.0 \mathrm{M}$ & $0,37 \mathrm{M}$ & $\mathrm{J} 1$ \\
\hline 20 & M.L.S.S & 53 & Costureira & Toxoplasmose ocular congênita & $6.0 \mathrm{M}$ & $0,37 \mathrm{M}$ & $\mathrm{J} 1$ \\
\hline 21 & T.S.R & 30 & Bancária & Nistamo Congênito & $4.0 \mathrm{M}$ & $0,37 \mathrm{M}$ & $\mathrm{J} 1$ \\
\hline 22 & W.L.O & 43 & Aposentado & Alta miopia & $8.0 \mathrm{M}$ & $0,37 \mathrm{M}$ & $\mathrm{J} 1$ \\
\hline 23 & W.J.S & 14 & Estudante & Catarata congênita & $6.0 \mathrm{M}$ & $0,37 \mathrm{M}$ & $\mathrm{J} 1$ \\
\hline 24 & L.M.Z & 80 & Aposentada & $\begin{array}{l}\text { Degeneração macular } \\
\text { Relacionada à idade }\end{array}$ & $6.0 \mathrm{M}$ & $0,37 \mathrm{M}$ & $\mathrm{J} 1$ \\
\hline 25 & J.N.B & 42 & Aposentado & Retinose pigmentária & $6.0 \mathrm{M}$ & $0,37 \mathrm{M}$ & $\mathrm{J} 1$ \\
\hline 26 & N.S.C & 23 & Estudante & Distrofia macular & $5.0 \mathrm{M}$ & $0,37 \mathrm{M}$ & $\mathrm{J} 1$ \\
\hline 27 & O.J.S & 53 & Aposentado & Retinopatia diabética & $8.0 \mathrm{M}$ & $0,37 \mathrm{M}$ & $\mathrm{J} 1$ \\
\hline 28 & C.A.B & 38 & Prendas Domésticas & Aniridia & $8.0 \mathrm{M}$ & $0,37 \mathrm{M}$ & $\mathrm{J} 1$ \\
\hline 29 & C.B & 18 & Universitário & Glaucoma congênito & $6.0 \mathrm{M}$ & $0,37 \mathrm{M}$ & $\mathrm{J} 1$ \\
\hline 30 & F.E.A & 22 & Analista de Sistemas & Atrofia do nervo óptico & $8.0 \mathrm{M}$ & $0,37 \mathrm{M}$ & $\mathrm{J} 1$ \\
\hline
\end{tabular}

magnificação têm bom manuseio e mobilidade, porém proporcionam aberração, redução de campo visual e distância focal muito próxima ${ }^{(7)}$.

O mercado dispõe de recursos eletrônicos portáteis como os importados Quickloock, Max, Max Lupe, entre outros. Além desses, o Alladin de fabricação nacional. Os recursos eletrônicos, CCTV e lupa eletrônica são excelentes alternativas para pessoas que necessitam de maior distância de trabalho, porque permitem ao paciente uma leitura com melhor contraste e resolução, distância focal e campo visual confortável, porém as di- mensões destes aparelhos impossibilitam a mobilidade e em alguns casos geram desconforto e náuseas quando utilizados por longo período ${ }^{(3,7)}$.

Seguindo critérios tecnológicos e de "design" para o desenvolvimento do SLP, o presente estudo propôs um recurso eletrônico portátil que supre as necessidades dos pacientes com baixa visão, garantindo mobilidade durante período mais longo de utilização e sem apresentar desconforto de leitura.

O número de letras e palavras que podem ser lidas no campo visual no momento de realizar a adaptação de 
auxílios ópticos é o grande desafio, para melhorar o aproveitamento do resíduo visual ${ }^{(8,9)}$. O SLP-permitiu a leitura de palavras com até oito letras simultaneamente com tamanho de fonte doze, possibilitando melhor visualização e reduzindo as perdas de linha de leitura.

Na rede pública de ensino fundamental, não há salas de recursos em quantidade suficiente e são poucas as bibliotecas universitárias e públicas equipadas com as tecnologias necessárias que permitam a acessibilidade do paciente com baixa visão ${ }^{(2,5)}$. Devido ao seu baixo peso, pode ser transportado em bolsas ou malas pequenas, garantindo a leitura das pessoas com baixa visão em locais públicos.

O SLP com seu "design" funcional, aliado às facilidades de manuseio e transporte, favorecerá o acesso à informação e ao estudo e beneficiará a população com baixa visão, principalmente os estudantes, proporcionando maior mobilidade, independência e inclusão social ${ }^{(10)}$.

Uma grande vantagem do SLP é o seu "design", que gerou manifestações auto relatadas de sensação emocional positiva e uma grande demonstração na vontade de utilização em locais públicos, escolas e bibliotecas; influência positiva na terapêutica e no estímulo de pessoas com deficiência visual. Observa-se que a não utilização de recursos ópticos por escolares adolescentes ou pré adolescentes pode estar relacionada a fatores psicológicos ${ }^{(2,3)}$.

\section{Conclusões}

O protótipo do SLP mostrou-se um recurso alternativo no processo de inclusão social das pessoas com baixa visão com diferentes níveis de resíduo visual. Também pode proporcionar incentivo psicológico, permitir conforto, mobilidade e independência àqueles que necessitam de uma leitura mais prolongada e maior distância de trabalho.

\section{Abstract}

Purpose: The proposal of this study is to test and to validate as the effectiveness and efficiency in a series of cases the prototype of a national equipment of magnification to reading. Methods: A recently developed of equipment of magnification (patent pending Brazilian Institutte of Industrial Property \# 020050145260) was tested in a group of 30 patients (age range 9 to 80 years, 17 males). A portable apparatus was developed with a system of capture of images coupled with a 5,6 inch monitor, providing an increase of 15 $X$. The effectiveness of the visual acuity and the reading efficiency were analyzed after the use of the proposed prototype. Results: Six patients $(20 \%)$ presented AV $8 M, 12$ patients (40\%) presented AV 6,0M, 7 patients $(23.3 \%)$ presented $A V 5 M, 5$ patients (16.7\%) presented $4 M$. The average of visual acuity before the utilization of SPR measured by LHNV-1 logMAR chart was 5,75M. After the use of LHNV-1 logMAR chart, 100\% (one hundred percent) of the patients reached the efficacy of $A V$ J1. Conclusion: The prototype of SPR is an alternative resource in the social inclusion process of low vision patients with different levels of visual residue. It demonstrates psychological incentive, allows comfort, mobility and independence to those who need a more extended lecture and more distance of work.

Keywords: Low vision/rehabilitation; Equipment design; Biomedical engineering; Reading; Quality oflife; Brazil

\section{RefERENCIAS}

1. Resnikoff S, Pascolini D, Etya'ale D, Kocur I, Pararajasegaram R, Pokharel GP, Mariotti SP. Global data on visual impairment in the year 2002. Bull World Health Organ. 2004;82(11):844-51.

2. Montilha RCI, Temporini ER, Nobre MIRS, Gasparetto MERF, Kara-José N. Utilização de recursos ópticos e equipamentos por escolares com deficiência visual. Arq Bras Oftalmol. 2006;69(2):207-11.

3. Harrison CM. Desing for people with low vision: learnability, usability and pleasurability. In: MCCabe PT, editor. Contemporary ergonomics. New York: CRC Press; 2004.

4. Castro CTM, Kallie CS, Salomão SR. Elaboração e validação de tabela MNREAD para o idioma português. Arq Bras Oftalmol. 2005;68(6):777-83.

5. Souza SC. Acessibilidade: Uma proposta de estrutura de serviços informacionais para usuários cegos e com visão subnormal em biblioteca universitária [tese]. Santa Catarina: Universidade Federal de Santa Catarina; 2004.

6. Meads C, Hyde C. What is cost of blindness? Br J Ophthalmol. 2003;87(10):1201-4.

7. Haddad MAO, Sampaio MW, Kara-José N. Auxílios para baixa visão. São Paulo: Laramara; 2001.

8. ABNT NBR 9050: 2004. Acessibilidade a edificações, mobiliário, espaços e equipamentos urbanos [Internet] 2004 Maio [citado 2007 Mar 19]: [cerca de 150p.]. Disponível em: http://www.mj.gov.br/sedh/ct/CORDE/dpdh/corde/ABNT/ NBR9050-31052004.pdf.

9. Beckmann PJ, Legge GE. Psychophysics of reading - XIV. The page navigation problem in using magnifiers. Vision Res.1996; 36(22):3723-33.

10. Torres EF, Mazzoni AA. Conteúdos digitais e multimídia: o foco na Usabilidade e Acessibilidade. Ci Inf. Brasília, 2004;33(2):152-60.

\section{Endereço para correspondência \\ Vagner Rogério dos Santos \\ Rua Noel José da Silva, no 170 - Penha \\ CEP 03678-030 - São Paulo (SP) - Brasil \\ fone: (11) 2046-8678 \\ e-mail: vagner_rogerio@yahoo.com.br}

